\title{
QUESTIONÁRIO PARA DIAGNÓSTICO DOS OBSTÁCULOS EM PEQUENAS E MÉDIAS EMPRESAS: ESTUDO VOLTADO AO RAMO DO TURISMO EM ADVENTO DA COVID-19
}

\author{
Higor Gibotti Carvalho \\ FATEC-SÃO CARLOS - CEETEPS, higor giboti@outlook.com \\ Ana Teresa Colenci Trevelin \\ Doutora, FATEC-SÃO CARLOS - CEETEPS, atcol@yahoo.com.br \\ Abner Santos Macedo \\ Mestrando, Universidade Federal de São Carlos. abnersantos.macedo@gmail.com
}

\begin{abstract}
RESUMO
Mediante os grandes transtornos ocasionados pela COVID-19 no mundo, o trade turístico sofreu severos impactos econômicos e financeiros. O presente artigo buscou na literatura elementos e variáveis que representam as principais dificuldades enfrentadas principalmente por pequenas e médias empresas. Através de uma revisão bibliográfica e revisão de escopo, realizadas nas bases Scopus, Scielo e Web of Science, utilizando-se descritores relacionados ao fenômeno estudado, algumas características que representam obstáculos, tais como, restrições governamentais, distanciamento social, adequação aos protocolos sanitários, necessidade de inserção ou aumento de presença digital e a necessidade de buscar habilidades e comportamentos empreendedores, foram analisadas e caracterizadas. Posteriormente, essas variáveis foram compiladas num questionário semiestruturado, validado por especialistas do turismo, principalmente sob a perspectiva de agências de turismo. Os principais resultados apontam para a busca por treinamentos especializados, por linhas de fomento como ação de contenção, aperfeiçoamento do modelo de gestão, principalmente nos aspectos de marketing, finanças e jurídico, sendo complementados por ajustes logísticos e operacionais como ações elementares para a retomada econômica. As principais limitações estão relacionadas a quantidade de bases analisadas, podendo ser expandidas, variações nos descritores também representam uma limitação e pelo tema ser incipiente. Como sugestão de futuros estudos, recomenda-se a aplicação do questionário desenvolvido, na modalidade survey, para diagnosticar a percepção dos impactos sob a perspectiva de diferentes stakeholders. $\mathrm{O}$ artigo contribui com o diagnóstico de importantes impactos econômicos e financeiros num dos setores mais afetados pela pandemia COVID-19, oferecendo insights e uma visão macro da situação atual.
\end{abstract}

Palavras-chave: Pandemia. Impactos. PMEs.

\section{ABSTRACT}

Due to the great inconveniences caused by COVID19 in the world, the tourist trade has suffered severe economic and financial impacts. This article searched the literature for elements and variables that represent the main difficulties faced mainly by small and medium- 
sized companies. Through a bibliographic review and scope review on Scopus, Scielo and Web of Science databases, using descriptors related to the studied phenomenon, some characteristics that represent obstacles, such as government restrictions, social distance, adequacy to sanitary protocols, need for insertion or increased digital presence and the need to seek entrepreneurial skills and behaviors were analyzed and characterized. These variables were compiled in a semi-structured questionnaire, validated by tourism specialists, mainly from the perspective of tourism agencies. The main results point to the search for specialized training, for promotion lines as a containment prevention, improvement of the management model, mainly in the aspects of marketing, finance and legal, being complemented by logistical and operational adjustments as elementary actions for the economic recovery. The limitations are related to the quantity of bases analyzed, variations in the descriptors also represent a limitation and the theme is incipient. As a suggestion for future studies, it is recommended to apply the questionnaire developed, in the survey mode, to diagnose the perception of impacts from the perspective of different stakeholders. The article contributes to the diagnosis of important economic and financial impacts in one of the sectors most affected by the COVID19 pandemic, offering insights and a macro view of the current situation.

Key-words: Pandemic. Impacts. SMEs.

\section{INTRODUÇÃO}

Negócios relacionados ao segmento turístico sofreram direta e negativamente os impactos causados pela pandemia COVID-19 desde seu reconhecimento, em março de 2020. A Organização Mundial do Turismo (OMT, 2020) declarou que a pandemia transformou 2020 no pior ano para o setor de turismo internacional. O segmento sofreu uma perda de 935 bilhões de dólares e uma redução de 900 milhões de turistas de janeiro a outubro de 2020.

No Brasil, segundo a Confederação Nacional de Municípios (CNM, 2019), o turismo corresponde a $8 \%$ do PIB. Estudos conduzidos pela Fundação Getúlio Vargas (FGV, 2020), estimam que o PIB em 2021 deverá recuar em torno de 4,2\% comparado a 2019, sendo projetado em torno de $\mathrm{R} \$ 259,4$ bilhões.

O relatório de inteligência do Serviço Brasileiro de Apoio às Micro e Pequenas Empresas (SEBRAE), voltado ao turismo, apresentou queda significativa nos indicadores do setor, visto que, em função da pandemia, diversas empresas ficaram impossibilitadas de abrirem suas portas e comercializarem produtos e serviços do trade (SEBRAE, 2020).

O relatório apresentou ainda os principais desafios para o setor, incluindo diversos stakeholders como, hotelaria, bares, empresas de eventos, atrações, agências e operadoras de viagem. Desemprego, cancelamentos de voos e hospedagens, retração econômica 


\section{Fatecnológica \\ Jahu}

ISSN 1980-8526 / ISSN 2763-5600

http://fatecjahu.edu.br/a-fatec-jahu/revista-fatecnologica/ email: fatecnologica.jahu@fatec.sp.gov.br

refletindo na queda do PIB, fechamento em massa de PMEs. São algumas características que ilustram o atual cenário (WTTC,2020; FGV, 2020; IGBE, 2020).

Nesse contexto é extremamente necessário que as empresas se reinventem para manter a competitividade e seu funcionamento. De acordo com o Sebrae (2020), em função nos aumentos das restrições de distanciamento social e quarentena, 31\%, ou seja, 5,3 milhões de pequenas empresas, mudaram o seu funcionamento e precisaram se adaptar para manter sua saúde financeira.

Ao aprofundar a análise, nota-se que dentre as empresas em funcionamento, 41,9\% realizam atualmente, apenas entregas online, 41,2\% trabalham com o horário reduzido e $21,6 \%$ atuam remotamente (SEBRAE, 2020).

Sendo assim, de acordo com Nilash et. Al (2021), os empreendedores precisarão se adaptar em diversas situações, incluindo: treinamentos e capacitações em marketing digital, finanças, prospecção e vendas, bem como em sua estrutura logística e operacional, tornando a necessidade de investimentos em treinamento e desenvolvimento, T\&D, extremamente relevante para manter a competitividade. Cada vez mais, o e-learning, mentorias, mobile learning, palestras via streaming e vídeo conferências vem ganhando um espaço importante (CNN BRASIL, 2020).

Essa pesquisa concentrou-se em analisar as principais dificuldades e obstáculos vivenciados pelas PMEs no atual cenário, considerando a COVID-19 e propor diretrizes que possam auxiliar na retomada do setor.

O objetivo dessa pesquisa foi realizar uma análise na literatura sobre os principais conceitos e características que devem compor um questionário semiestruturado, para diagnosticar as principais demandas existentes atualmente em PMEs do segmento do turismo, mediante aos grandes impactos negativos resultantes da pandemia COVID-19.

Para buscar as respostas, o estudo se baseou na seguinte questão de pesquisa: Quais são as principais variáveis que representam obstáculos em PMEs do trade turístico? Quais estratégias podem afetar positivamente no setor de turismo que sofre com a queda gerada pela COVID -19?

Primeiramente foi realizada uma revisão de escopo com o objetivo de buscar na literatura as principais variáveis que afetam as PMEs, seguida por uma revisão bibliográfica nas bases de dados Scopus, Web of Science e Scielo, em busca de levantar as principais 


\section{Fateç chu}

ISSN 1980-8526 / ISSN 2763-5600

http://fatecjahu.edu.br/a-fatec-jahu/revista-fatecnologica/ email: fatecnologica.jahu@fatec.sp.gov.br

dificuldades enfrentadas no turismo nos últimos cinco anos, no entanto, com ênfase nas publicações de 2020 e 2021.

Posteriormente os resultados foram categorizados e sumarizados num questionário e validado por três especialistas, representando empresas do segmento devidamente registradas no CADASTUR (sistema de cadastro de pessoas físicas e jurídicas que atuam no setor do turismo) tornando-o exequível em futuros estudos.

\section{MATERIAIS E MÉTODOS}

Para fazer uma análise do estado da arte sobre o tema dificuldades no turismo em PMEs do trade, buscando auxiliar na retomada do turismo pós-pandemia, apesar de existirem inúmeras empresas que compõem o segmento do turismo, este estudo se restringe a analisar a problemática sobre a perspectiva das empresas que realizam agenciamento de viagens, versado nesta pesquisa, foi realizada inicialmente uma revisão bibliográfica tradicional, seguida de uma revisão de escopo e posteriormente a elaboração de um questionário semiestruturado, com base nos resultados obtidos nas revisões.

O objetivo da revisão bibliográfica tradicional é analisar perante a literatura quais são as principais fontes, teorias, conceitos e ideias acerca de um determinado tema. Para Jesson et al. (2011) os principais fundamentos epistemológicos, ontológicos, a identificação das principais questões e problemas que foram abordados até o momento, podem ser identificados através da revisão bibliográfica. Dessa forma, entende-se que a revisão direciona as principais questões e debates sobre o tema. A Figura 1 ilustra as etapas utilizadas na condução do artigo.

Figura 1. Fases e condução da pesquisa

\section{Revisão bibliográfica}

- Análise do estado da arte

\section{Revisão de escopo}

- Levantamento das principais características do fenômeno

\section{Elaboração do questionário}

- Contendo as principais características encontradas na literatura e através do conhecimento empírico

Fonte: Autores (2021). 


\section{Fatecnológica \\ Jahu}

ISSN 1980-8526 / ISSN 2763-5600

http://fatecjahu.edu.br/a-fatec-jahu/revista-fatecnologica/ email: fatecnologica.jahu@fatec.sp.gov.br

Quais são essas barreiras? Para buscar respostas, em ambas as bases, foram utilizados os seguintes descritores: (tourism) and (pandemic) and (covid or corona) and (sme).

As bases selecionadas nesse artigo para realizar a busca do referencial teórico foram: a Scielo, Scopus e Web of Science (Derwent Innovations Index, $\mathrm{KCl}$, Russian Science Citation Index e Scielo Citation Index).

Como fator determinante na seleção das bases, reconheceu-se a importância de se explorar artigos relacionados as áreas de turismo, educacionais ou relacionados à área de gestão, com ênfase em gestão de pessoas. Vale destacar que na busca, foram considerados apenas artigos de 2016 a 2021.

Os resultados preliminares obtidos foram: Scielo - 0 artigos; Scopus - 117 artigos e Web of Science - 2 artigos, totalizando 119 artigos. Primeiro realizou-se a leitura de título, resumo e palavras chaves de cada artigo. Posteriormente, foi realizada a leitura de introdução e conclusão. Ao final, filtrando-se apenas artigos convergentes com o tema discorrido nesta pesquisa, realizou-se a leitura e fichamento de 63 artigos.

Nesse contexto, considera-se a abordagem dessa pesquisa qualitativa, exploratória e descritiva. Gil (2008) define uma pesquisa exploratória como aquela que busca explicitar um problema, ou seja, proporcionar maior familiaridade. O autor classifica uma pesquisa descritiva como aquela que descreve as características de uma determinada população.

Já a revisão de escopo, de acordo com Arksey e O’Malley (2005) focam em mapear de maneira ágil os conceitos elementares que permeiam uma determinada área de pesquisa. O método difere de uma revisão bibliográfica tradicional pois envolve uma pesquisa mais sistematizada, mas não chega a ser uma revisão sistemática, por não visar avaliar a qualidade das evidências disponíveis.

No tocante a condução da revisão de escopo, a Figura 2 apresenta descreve as etapas que foram implementadas através do protocolo estabelecido previamente.

Após as revisões bibliográficas e de escopo, coletando-se as principais demandas e diagnosticando ações que objetivam mitigar os problemas chave enfrentados pelo turismo, o foco foi converter os resultados em questões a serem respondidas por gestores de agências de turismo. 
Figura 2. Protocolo da revisão de escopo

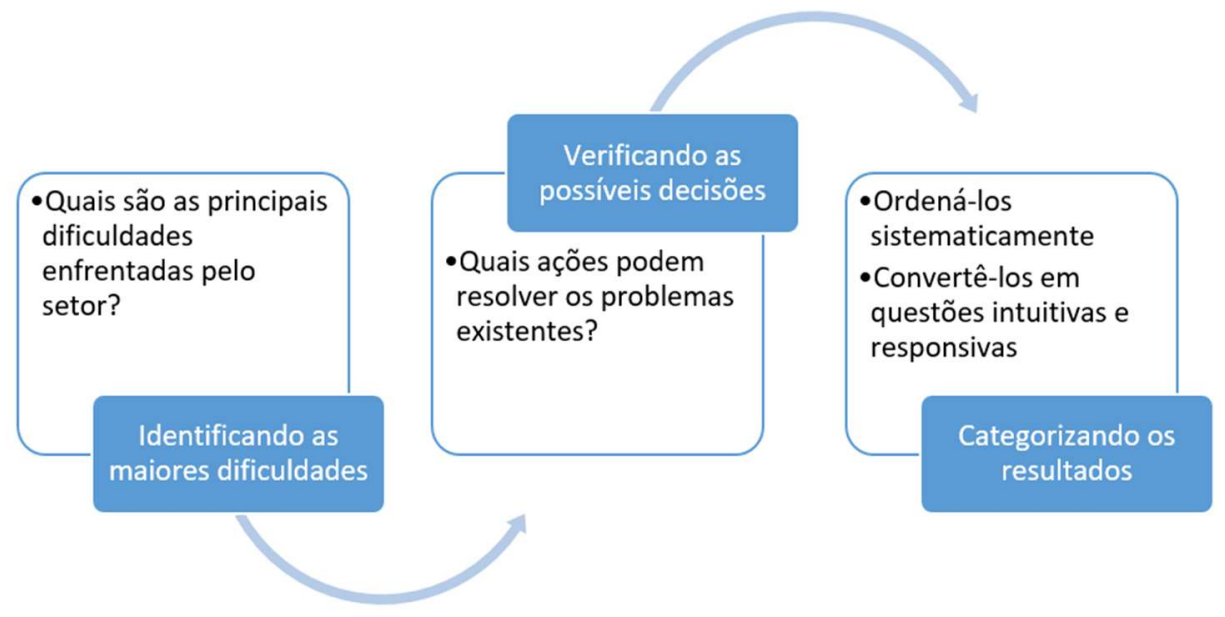

Fonte: Autores (2021).

A utilização da entrevista semiestruturada assegura diversas vantagens à pesquisa, tais como, a possibilidade de ser utilizada em diversos segmentos da população, promover maior flexibilidade, visto que, o entrevistador pode repetir a pergunta, formulá-la de maneira diferente até que haja garantia de que foi compreendido, proporciona informações mais precisas e permite que os dados (MARCONI; LAKATOS, 1999, P. 94).

Optou-se por questões alternativas, respostas variando de 1 a 5, onde 1 corresponde a discordo totalmente e 5 a concordo totalmente. Como parâmetro a escala Likert, que é comumente utilizada na ciência para mensurar atitudes e comportamentos, capaz de extrair informações qualitativas de uma pergunta estruturada de forma quantitativa (ALBAUM, 1997).

Após a elaboração do questionário, veio a etapa de validação. Para validar, foram selecionadas três agências de turismo, devidamente cadastradas no CADASTUR, estabelecidas desde o momento antes da pandemia até o presente momento.

A validação foi feita por entrevistas, através de canais digitais e com instruções claras de preenchimento e acompanhamento simultâneo. Algumas alterações foram sugeridas e após a sua realização, foi validado com mais duas agências também devidamente cadastradas.

Concluindo-se a etapa de validação, os questionários tornaram-se aptos a serem submetidos através de uma survey para uma população e/ou amostra mais abrangente e ampla. 


\section{RESULTADOS E DISCUSSÃO}

Ao utilizar os descritores de buscas definidos previamente, na base de dados Web of Science, incluindo em sua totalidade as bases: Derwent Innovations Index, $\mathrm{KCl}$, Russian Science Citation Index e Scielo Citation Index, foi possível encontrar apenas dois artigos publicados sobre o tema.

Ao utilizar os descritores de buscas definidos previamente, na base de dados Scopus, foram encontradas 117 ocorrências. Filtrando-se os artigos através da leitura de títulos, resumos e palavras chaves, foram excluídos 54 artigos que não correspondiam ao tema e fenômeno pesquisado, restando 63 para análise. A Figura 3 apresenta a relação de publicação por países.

Figura 3. Países que mais publicam sobre o tema

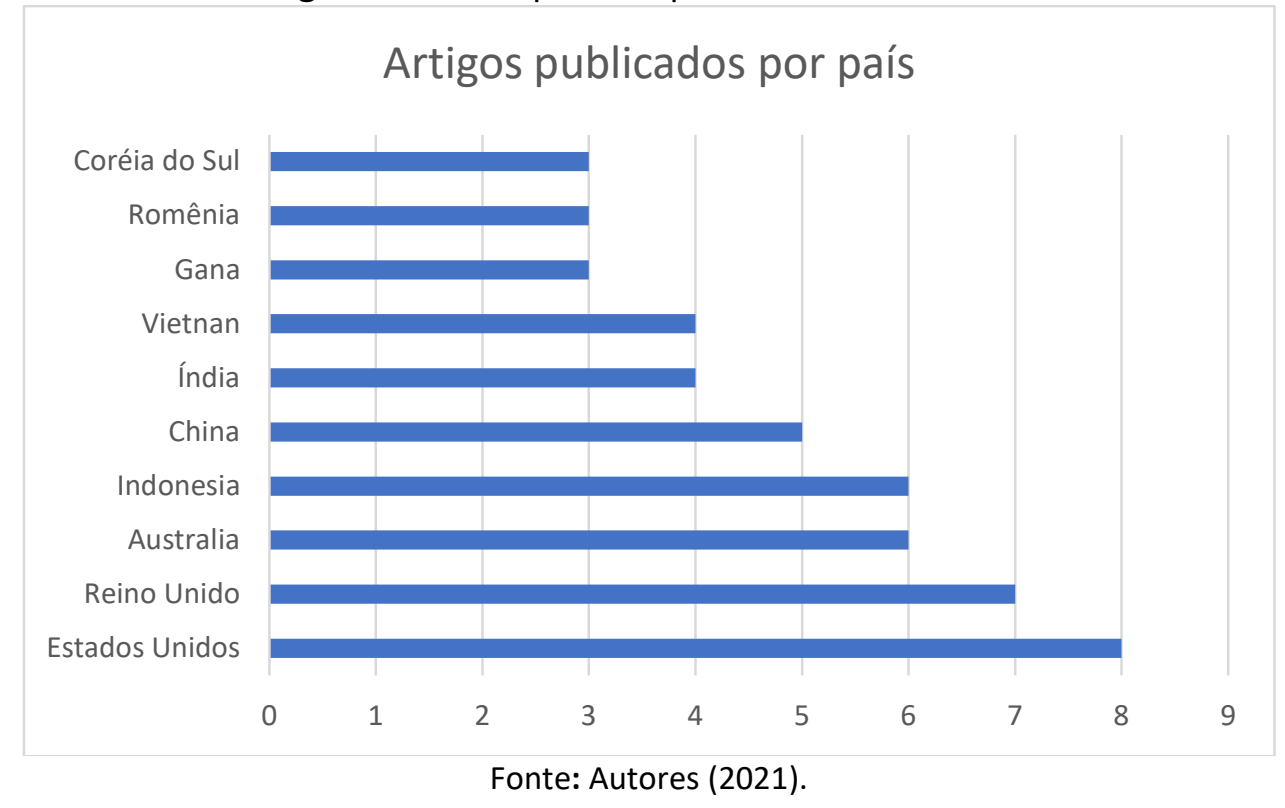

Considerando as buscas na Scopus, por exemplo, nota-se que os dez países que mais publicaram acerca dos efeitos da pandemia em PMEs atuantes no trade turístico foram respectivamente: Estados Unidos (8 artigos), Reino Unido (7 artigos), Austrália (6 artigos), Indonésia (6 artigos), China (5 artigos), Índia (4 artigos), Vietnam (4 artigos), Gana, Romênia e Coréia do Sul (3 artigos cada).

Os autores que mais publicaram artigos são: Kim, W.G., Kristinae, V., Lu, L., Peng, J., Ratten. V., Wu, J., com duas publicações cada e, Abdelazis, A.S., Acquaah, M., Aduhene, D.t. e Agareal, R.N. como uma publicação de cada autor. 
Os cinco principais periódicos que publicam sobre o tema são: International Journal of Hospitality Management (11 artigos), Susteinability Swtizerland (10 artigos), Tourism Management ( 3 artigos), Anatolia e Strategic Change com 2 artigos cada.

Com relação a relevância do tema, fica evidente a ascensão do debate em âmbito acadêmico, visto que, em 2020 foram publicados 28 artigos e, em 2021 até o presente momento, foram publicados 35 artigos.

Figura 4. Quantidade de periódicos e respectivos fatores de impacto.

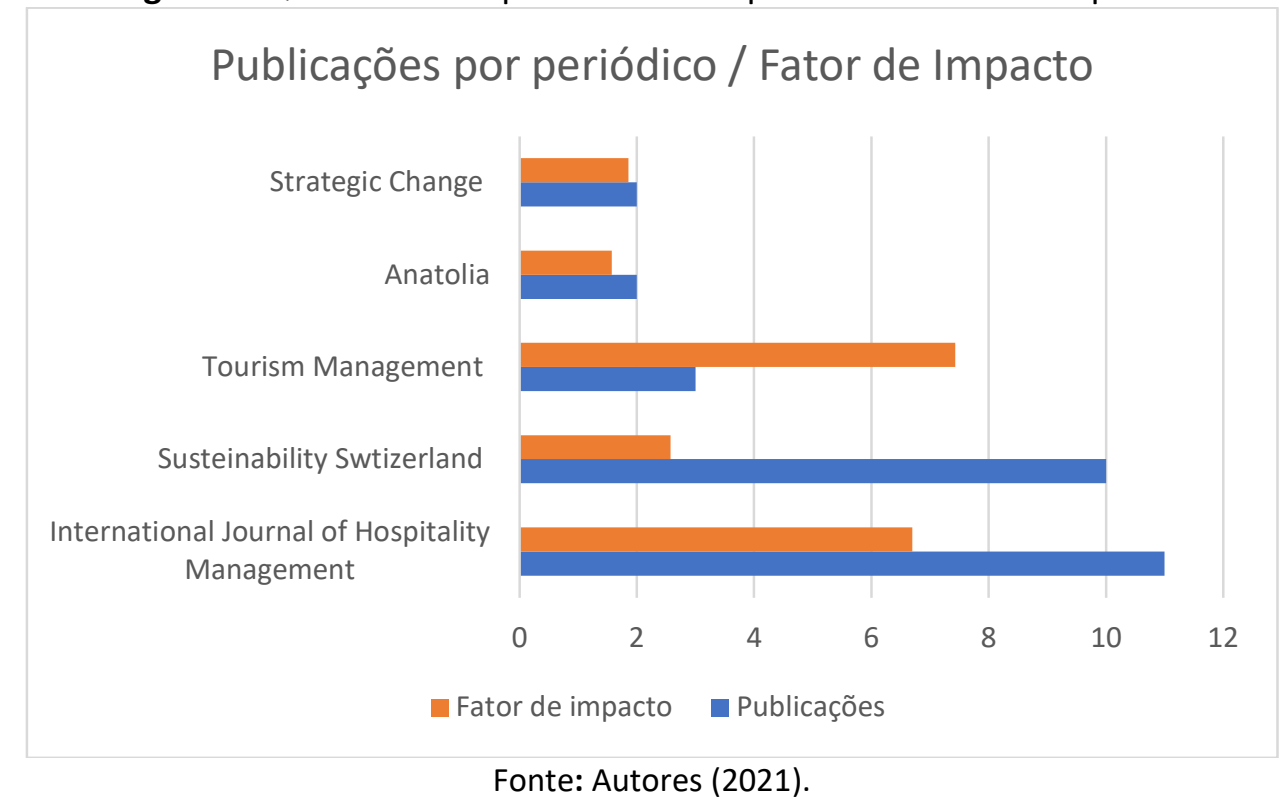

Os principais resultados encontrados em ambas as bases de dados foram analisados, categorizados e apresentados na Tabela 1. A tabela sumariza os artigos que auxiliaram no diagnóstico das principais lacunas, ou seja, dificuldades enfrentadas pelas PMEs, em decorrência da COVID-19.

Tabela 1. Principais artigos encontrados apresentando características que afetam o turismo na atual conjuntura pandêmica.

\begin{tabular}{|l|c|c|}
\hline \multicolumn{1}{|c|}{ Trabalhos relevantes } & Problemas & Possíveis soluções \\
\hline Itani, O.S., Hollebeek, L.D. (2021) & Distanciamento social & Realidade virtual \\
\hline Thukral, E. (2021); Sobaih, A.E. E. et al. & Intervenções & Resiliência \\
(2021); Gregurec, I. et al. (2021); Al- & governamentais & \\
Fadly, Ahmad (2020); Kukanja, M; & & \\
Planinc, T; Sikosek, M (2020). & & \\
\hline Panfluk, E. (2021); Buijtendijk, H. et al. & Impactos econômicos & \\
(2021); Pascual-Fernández, P. et al. & & \\
(2021); Vig, S. Agarwal, R. N. (2021); & & \\
Aduhene, D. T, Osei-Assibey, E. (2021); & & \\
Gregurec, I. et al. (2021); Al-Fadly, & & \\
Ahmad (2020); Kukanja, M; Planinc, T; & & \\
Sikosek, M (2020). & & \\
\hline
\end{tabular}




\begin{tabular}{|c|c|c|}
\hline $\begin{array}{l}\text { Nilashi, M. et al. (2021); Ahn, J., Know, J. } \\
\text { (2021); }\end{array}$ & $\begin{array}{l}\text { Tomada de decisão dos } \\
\text { turistas }\end{array}$ & $\begin{array}{l}\text { Mídias sociais } \\
\text { Sistemas de } \\
\text { recomendações }\end{array}$ \\
\hline $\begin{array}{l}\text { Leta, S. D., Chan, I. C. C. (2021); Le, D., } \\
\text { Phi, G. (2021); Pillai, S. G. et al. (2021); } \\
\text { Rivera, M. et al. (2021); }\end{array}$ & $\begin{array}{l}\text { Redução na procura por } \\
\text { hospedagens }\end{array}$ & $\begin{array}{l}\text { Investimentos em } \\
\text { gerenciamento de crises }\end{array}$ \\
\hline $\begin{array}{l}\text { Untaru, E. -N., Han, H. (2021); Magnini, } \\
\text { V. P., Zehrer, A. (2021); }\end{array}$ & Crise sanitária & $\begin{array}{l}\text { Medidas de proteção e } \\
\text { rígidos protocolos de } \\
\text { higiene }\end{array}$ \\
\hline $\begin{array}{l}\text { Wieczorek-Kosmala, M. (2021); Rivera, } \\
\text { M. et al. (2021); Pascual-Fernández, P. } \\
\text { et al. (2021); Vig, S. Agarwal, R. N. } \\
\text { (2021); }\end{array}$ & Problemas financeiros & $\begin{array}{l}\text { Estratégias de contenção e } \\
\text { prevenção (Fluxo de } \\
\text { Caixa) }\end{array}$ \\
\hline $\begin{array}{l}\text { Pascual-Fernández, P. et al. (2021); } \\
\text { Kukanja, M; Planinc, T; Sikosek, M } \\
(2020) \text {. }\end{array}$ & $\begin{array}{l}\text { Necessidade de projeção } \\
\text { de vendas e } \\
\text { comercialização de } \\
\text { serviços/produtos }\end{array}$ & Marketing \\
\hline $\begin{array}{l}\text { Lu, L. et al. (2021); Al-Fadly, Ahmad } \\
\text { (2020); Kukanja, M; Planinc, T; Sikosek, } \\
\text { M (2020). }\end{array}$ & Gestão e logística & Serviços online \\
\hline $\begin{array}{l}\text { Ratten, V., Jones, P. (2021); Li, Z. et al. } \\
\text { (2021); Al-Fadly, Ahmad (2020); }\end{array}$ & $\begin{array}{c}\text { Ausência de habilidades e } \\
\text { competências } \\
\text { empreendedoras }\end{array}$ & $\begin{array}{c}\text { Cursos voltados a } \\
\text { educação e orientação } \\
\text { empreendedora }\end{array}$ \\
\hline
\end{tabular}

Fonte: Autores (2021).

A pesquisa apontou para diversas variáveis que interferem negativamente no turismo em detrimento ao atual cenário pandêmico global. A literatura, apesar de recente, detalha problemas pontuais abrangentes e complexos, que demanda amplo estudo e aprofundamento nas análises para viabilizar soluções inteligentes (AL-FADLY, AHMAD, 2020).

A busca demonstrou cientificamente, de acordo com (Kukanja, Planinc e Sikosek (2020) que as PMEs estão sofrendo impactos extremamente negativos, principalmente em função das restrições governamentais, mediante a necessidade de distanciamento social, ocasionando queda na geração de receita, levando milhares de negócios ao encerramento de suas atividades no Brasil.

Este cenário abala a confiança do turista, resultando em quedas drásticas na geração de renda principalmente de estabelecimentos turísticos relacionados a hospedagem, agenciamento, transporte e prestação de serviços turísticos. Complementarmente, a necessidade de adequações sanitárias, para seguir os rígidos protocolos exigidos pelas entidades regulamentadoras, demandam grandes investimentos, dificultando ainda mais a capacidade operacional e logística de PMEs, num momento de retração financeira (THUKRAL; SOBAIH ET AL.; GREGUREC ET AL. 2021; AL-FADLY; AHMAD; KUKANJA; PLANINC; SOKOSEK, 2020). 
A complexidade aumenta ainda mais quando além de todas as dificuldades supracitadas, são somadas a ausência de habilidades e competências empreendedoras, demandadas neste momento de crise, dificultam a adequação a protocolos e a gestão logística e operacional, principalmente em PMEs que necessitam de transformações digitais consideráveis (RATTEN; JONES; LI ET AL, 2021).

O questionário resultante da pesquisa é apresentado no Apêndice A. Foi aplicado, alterado e validado por três agências de turismo devidamente cadastrada no CADASTUR, tornando-se apto a aplicações exploratórias em futuros estudos.

\section{CONSIDERAÇÕES FINAIS}

A literatura aponta para a necessidade de PMEs realizarem treinamentos e capacitações em gestão, empreendedorismo, marketing digital e finanças como formas de reduzir os impactos existentes na atualidade no setor do turismo. A busca por linhas de crédito e ações de fomento, são alternativas para manter a saúde financeira e a competitividade.

O estudo apresenta limitações por explorar um tema recente, tornando as buscas incipientes. O fato da unidade de analise ter sido restringida as empresas que realizam agenciamento de viagens, também é uma limitação, sendo possível em futuros estudos explorar a perspectiva de outras empresas e outros players do segmento. A quantidade de bases de dados foi limitada, podendo ser ampliadas para uma cobertura mais completa.

Como sugestão para futuros estudos, recomenda-se uma aplicação de questionários no trade turístico para diagnosticar de forma prática as dificuldades enfrentadas, corroborando ou refutando as evidências apresentadas nesse trabalho, encontradas na literatura. O artigo contribui com o diagnóstico de importantes impactos econômicos e financeiros num dos setores mais afetados, de maneira severa, pela pandemia COVID-19.

\section{REFERÊNCIAS}

AGÊNCIA BRASIL. Crise do coronavírus já custa R\$ 3,9 bilhões a operadoras de turismo. Disponível em: https://agenciabrasil.ebc.com.br/geral/noticia/2020-04/crise-docoronavirus-ja-custa-r-39-bilhoes-operadoras-de-turismo. Acesso em: 15 mar. 2021.

ALBAUM, Gerald. The Likert Sacale Revisited. International Journal of Market Research. V. 39, n. 2, p. 331-348. Mar, 1997. 
AL-FADLY, Ahmad. Impact of Covid-19 on smes and employment. Entrepreneurshio and sustainability issue. V.8, n.2, p.629-648, dez./2020.

Arksey H, O'Malley L. Scoping studies: towards a methodological framework. Int. j. soc. res. methodol. 2005; 8:19-32.

CHIAVENATO, Idalberto. Gestão de pessoas: e o novo papel dos recursos humanos nas organizações. ed.7. Rio de Janeiro: Elsevier, 2010.

CHIAVENATO, Idalberto. Treinamento e desenvolvimento de recursos humanos: como incrementar talentos na empresa. Ed.7, São Paulo: Atlas, 2008.

CONFEDERAÇÃO NACIONAL DE MUNICÍPIOS. Turismo é responsável por $\mathbf{8 \%}$ da economia, participação no PIB cresce. Disponível em:

https://www.cnm.org.br/comunicacao/noticias/turismo-e-responsavel-por-8-da-economiaparticipacao-no-pib-cresce. Acesso em: 15 mar. 2021.

FGV. Impactos Econômicos da COVID-19 propostas para o turismo. Disponível em: https://fgvprojetos.fgv.br/sites/fgvprojetos.fgv.br/files/02.covid19_impactoeconomico_turis mo2_v07_fichacatolografica.pdf. Acesso em: 18 mar. 2021.

GIL, Antonio Carlos. Como elaborar projetos de pesquisa. 4. ed. São Paulo: Atlas, 2008.

IBGE. INDICADORES CONJUNTURAIS - COVID-19. Disponível em: https://agenciadenoticias.ibge.gov.br/media/com_mediaibge/arquivos/548281f191c80ecbb b69846b0d745eb5,pdf. Acesso em: 21 mar. 2021.

JESSON, J.K.; MATHESON, L.; LACEY, F.M. Doing your Literature Review: Traditional and Systematic Techniques. Sage Publications Ltd, 2011. Necessidades e provisão de treinamento para PMEs de turismo e hospitalidade: uma análise sub-regional.

KUKANJA et al. Crisis Management Practices in Tourism SMEs During the Covid-19 Pandemic. Organizacija, Slovenia, V.53, n. 4, p. 346-361, dez/2020. Disponível em: http://organizacija.fov.uni-mb.si/index.php/organizacija/article/view/1391. Acesso em: 25 mar. 2021.

MARCONI, Marina de Andrade; LAKATOS, Eva Maria. Técnicas de pesquisa. 4. ed. São Paulo: Atlas, 1999.

MARRAS, J.P. Administração de recursos humanos: do operacional ao estratégico. 13. ed., São Paulo. Saraiva, 2009.

MAYAKAA, Melphon; Jhon S. Akama. Systems approach to tourism training and education. Tourism Managenebt, v. 28. N. 1, p. 298-306, Fev. 2007. 
MILKOVICH, George T. Administração de Recursos Humanos: Treinamento. São Paulo: Editora Atlas, 2000.

ONU NEWS. Pandemia transforma 2020 no pior ano para o setor de turismo internacional. Disponível em: https://news.un.org/pt/story/2020/12/1736672. Acesso em: 10 mar. 2021.

SEBRAE. 0 impacto da pandemia de coronavirus nos pequenos negócios. Disponível em: https://www.sebrae.com.br/sites/PortalSebrae/artigos/o-impacto-da-pandemia-decoronavirus-nos-pequenos-negocios,192da538c1be1710VgnVCM1000004c00210aRCRD. Acesso em: 15 mar. 2021.

SEBRAE. Relatório de inteligência: impactos da pandemia da covid19 sobre o turismo. Disponível em: https://atendimento.sebrae-sc.com.br/inteligencia/relatorio-deinteligencia/impactos-da-pandemia-da-covid-19-sobre-o-turismo. Acesso em: 15 mar. 2021.

“Os autores declaram estar cientes quanto a responsabilidade pelo conteúdo do artigo." 


\section{Fatecnológica}

ISSN 1980-8526 / ISSN 2763-5600 http://fatecjahu.edu.br/a-fatec-jahu/revista-fatecnologica/ email: fatecnologica.jahu@fatec.sp.gov.br

APÊNDICE A

\begin{tabular}{|c|c|c|c|c|c|}
\hline QUESTIONÁRIO - AGÊNCIAS DE TURISMO & $\begin{array}{l}\text { Discordo } \\
\text { totalmente }\end{array}$ & Discordo & Neutro & Concordo & $\begin{array}{l}\text { Concordo } \\
\text { totalmente }\end{array}$ \\
\hline \multicolumn{6}{|l|}{ Barreiras relacionadas as características econômicas/financeiras } \\
\hline \multicolumn{6}{|l|}{$\begin{array}{l}\text { As restrições governamentais em decorrência da COVID19 afetaram negativamente, de maneira } \\
\text { significativa as agências de turismo no contexto financeiro. }\end{array}$} \\
\hline \multicolumn{6}{|l|}{$\begin{array}{l}\text { A pandemia provocou cancelamentos de voos e remarcações, aumentando pedidos de reembolsos } \\
\text { prejudicando as agências de turismo e outros steakholders do trade. }\end{array}$} \\
\hline \multicolumn{6}{|l|}{$\begin{array}{l}\text { As regras de distanciamento social e aumento no rigor das normas sanitárias inviabilizaram } \\
\text { funcionamento de lojas físicas de agências de turismo. }\end{array}$} \\
\hline \multicolumn{6}{|l|}{$\begin{array}{l}\text { A mudança provocada pela pandemia, alterou os critérios de decisão na hora de contratação feita pelo } \\
\text { turista, interferindo nas vendas e consequentemente nas receitas geradas por agências de turismo. }\end{array}$} \\
\hline \multicolumn{6}{|l|}{ A baixa procura por hospedagens impacta negativamente nas vendas de agências de turismo. } \\
\hline \multicolumn{6}{|l|}{ A baixa procura por passagens aéreas impacta negativamente nas vendas de agências de turismo. } \\
\hline \multicolumn{6}{|l|}{ A baixa procura por passagens terrestres impacta negativamente nas vendas de agências de turismo. } \\
\hline \multicolumn{6}{|l|}{ O índice de demissões aumentou em função da pandemia. } \\
\hline \multicolumn{6}{|l|}{ A saúde mental dos integrantes da empresa foi comprometida pela pandemia. } \\
\hline \multicolumn{6}{|l|}{ Barreiras relacionadas as características empreendedoras } \\
\hline \multicolumn{6}{|l|}{$\begin{array}{l}\text { As restrições de funcionamento ampliaram a necessidade de construir ou ampliar uma presença } \\
\text { digital de pequenas e médias empresas do setor do turismo. }\end{array}$} \\
\hline \multicolumn{6}{|l|}{$\begin{array}{l}\text { Com as constantes alterações no funcionamento das empresas, existe a necessidade constante da } \\
\text { busca por treinamentos e capacitações. }\end{array}$} \\
\hline \multicolumn{6}{|l|}{$\begin{array}{l}\text { As empresas precisam construir ou ampliar a presença digital para manterem-se competitivas e em } \\
\text { funcionamento. }\end{array}$} \\
\hline \multicolumn{6}{|l|}{ Em função da pandemia as empresas precisam alterar seu modelo de negócio constantemente. } \\
\hline \multicolumn{6}{|l|}{ Em função da pandemia as empresas precisam alterar sua logística de funcionamento constantemente. } \\
\hline \multicolumn{6}{|l|}{$\begin{array}{l}\text { Grande parte dos problemas enfrentados estão relacionados ás finanças da empresa, demandando } \\
\text { treinamento e capacitação continuamente. }\end{array}$} \\
\hline $\begin{array}{l}\text { Grande parte dos problemas enfrentados estão relacionados o marketing digital, demandando } \\
\text { treinamento e capacitação continuamente. }\end{array}$ & & & & & \\
\hline
\end{tabular}

Fatecnológica | Jahu, SP | v. 15 | n. 1 | p. 79-92 | 2021 | ISSN 1980-8526 (versão PDF-impressa) | ISSN 2763-5600 (versão online) 


\section{Fatecnológica

Inovar e estar atualizado são formas de se reinventar e manter-se competitivo.

Estar atualizado continuamente com novas publicações nos diários oficiais é extremamente necessário para manter as empresas em funcionamento adequadamente.

\section{T\&D como redutor dos impactos no turismo}

Atualizar-se através de cursos e capacitações podem mitigar os efeitos da pandemia.

Capacitações em gestão são fundamentais no enfrentamento aos efeitos da pandemia.

Capacitações em marketing são fundamentais no enfrentamento aos efeitos da pandemia.

Capacitações em finanças são fundamentais no enfrentamento aos efeitos da pandemia.

Capacitações em vendas são fundamentais no enfrentamento aos efeitos da pandemia.

Capacitações em como acessar linhas de crédito para fomento são fundamentais no enfrentamento aos efeitos da pandemia.

Capacitações em como acessar linhas de crédito para fomento são fundamentais no enfrentamento aos efeitos da pandemia.

Capacitações jurídicas com foco em resolução de conflitos são fundamentais no enfrentamento aos efeitos da pandemia.

Treinamentos com cunho psicológico são fundamentais para manter a produtividade e motivação da equipe.

Plataformas digitais e recursos tecnológicos são essenciais para mitigar os efeitos da pandemia. 